\title{
A numerical study of Bi-periodic binary diffraction gratings for solar cell applications
}

\author{
A. Mellor*, I. Tobías, A. Martí, A. Luque \\ Instituto de Energía Solar, Universidad Politécnica de Madrid, Madrid 28040, Spain
}

\begin{abstract}
A B S T R A C T
In this paper, a numerical study is made of simple bi-periodic binary diffraction gratings for solar cell applications. The gratings consist of hexagonal arrays of elliptical towers and wells etched directly into the solar cell substrate. The gratings are applied to two distinct solar cell technologies: a quantum dot intermediate band solar cell (QD-IBSC) and a crystalline silicon solar cell (SSC). In each case, the expected photocurrent increase due to the presence of the grating is calculated assuming AM1.5D illumination. For each technology, the grating period, well/tower depth and well/tower radii are optimised to maximise the photocurrent. The optimum parameters are presented. Results are presented for QD-IBSCs with a range of quantum dot layers and for SSCs with a range of thicknesses. For the QD-IBSC, it is found that the optimised grating leads to an absorption enhancement above that calculated for an ideally Lambertian scatterer for cells with less than 70 quantum dot layers. In a QD-IBSC with 50 quantum dot layers equipped with the optimum grating, the weak intermediate band to conduction band transition absorbs roughly half the photons in the corresponding sub-range of the AM1.5D spectrum. For the SSC, it is found that the optimised grating leads to an absorption enhancement above that calculated for an ideally Lambertian scatterer for cells with thicknesses of $10 \mu \mathrm{m}$ or greater. A $20 \mu \mathrm{m}$ thick SSC equipped with the optimised grating leads to an absorption enhancement above that of a $200 \mu \mathrm{m}$ thick SSC equipped with a planar back reflector.
\end{abstract}

Keywords:

Diffraction grating

Absorption enhancement

Light trapping

Surface texturing

Intermediate band solar cell

\section{Introduction}

Solar cells that absorb part of the solar spectrum weakly require absorption enhancement for efficient energy conversion. This can be achieved by light trapping, one means of which is application of a diffraction grating to either face of the solar cell [1-5]. The diffraction grating allows incident light to be coupled to internal orders whose wavevector lateral component is greater than those of the set of incident wavevectors. In a planar structure, these orders exist but are unexcited by the incident illumination. The orders excited in the presence of the grating are, in general, more numerous and have a higher path length inside the solar cell than those excited in a planar structure. Their excitation in the presence of the grating therefore leads to absorption enhancement. Diffraction gratings applied to the front surface of the solar cell can have the added benefit of reducing front surface reflection. However in this paper we focus on the properties of backside diffraction gratings.

Previous work has shown that diffraction gratings are theoretically capable of far greater absorption enhancement than the so called Lambertian scatterers, and that bi-periodic gratings are capable of far greater absorption enhancement than uni-periodic gratings [6]. Simple bi-periodic gratings can be fabricated on the nano-scale using lithographic techniques [7]. Nano-imprinting techniques allow such structures to be fabricated with high throughput [8], making future industrialisation a possibility.

In this paper, computational results are presented of absorption enhancement due to bi-periodic binary gratings consisting of hexagonal arrays of elliptical wells and towers. Studies are made of absorption enhancement in a quantum dot intermediate band solar cell (QD-IBSC) and in a thin crystalline silicon solar cell (SSC). The grating parameters are optimised and comparison is made to absorption enhancement using an ideally Lambertian scatterer.

\section{The simulation method, simulated structure and conditions}

\subsection{Simulation method}

A common means of calculating the absorption enhancement in a solar cell equipped with a diffraction grating is to apply Rigorous Coupled Wave Analysis (RCWA) to the entire structure: cell and grating $[3,4,9]$. Our approach is to simulate the grating and solar cell separately. The grating's scattering matrix is 
calculated using RCWA, and a separate matrix is generated analytically to describe the cell. A rigorous description of this formalism is described in [6], and a general description of the RCWA method can be found in [10]. The RCWA calculations are implemented using commercial software package Gd-Calc ${ }^{\circledR}$. The advantage of our simulation technique is that the same grating can be applied to cells with different absorption profiles without a computationally costly re-simulation of the grating, and that the period can be optimised without re-simulating the grating for each period tested (see section 3.1).

In this paper the simulation method will be applied to the optimisation of bi-periodic binary gratings for application in two distinct cell types; both of which are thicker than the coherence length of the incident solar radiation ( $\approx 1 \mu \mathrm{m}$ ), yet require light trapping. It should be noted that, for these cells, internal diffraction orders are temporally incoherent with one another. The time average of interference effects between orders should therefore be null [11]. Straightforward simulation of the entire cell structures with RCWA assumes temporal coherence and hence produces interference effects, which, for these cells, is unphysical. Some authors have overcome this by varying the thickness slightly over many simulations and averaging to reduce interference effects to negligible levels [9], albeit with added computational cost. With our technique, temporal incoherence between internal orders is assumed within a single simulation, increasing computational efficiency. The drawback of our technique is that it is only applicable to solar cells whose thickness is greater than the coherence length, although we believe with some effort it could be altered to account for the interference effects that are important in thin film devices.

The numerical calculation takes as input the grating geometry and profile, the incident wavelength $\lambda$, the absorption coefficient $\alpha(\lambda)$ and absorbing layer width $w$ and outputs the fraction of incident photons absorbed $A(\lambda)$. The photogenerated current is calculated from

$J_{p h}=q_{e} \int \Phi_{A m 1.5 D}(\lambda) A(\lambda) d \lambda$

where $q_{e}$ is the elementary charge and $\Phi_{A m 1.5 D}(\lambda)$ is the incident AM1.5D photon flux. The product of absorption coefficient and absorbing layer width $\alpha w$ shall hereafter be referred to as the normal absorbance.

\subsection{Cell types}

Absorption enhancement will be studied for cell types with distinct absorption profiles: a $40 \mu \mathrm{m}$ thick crystalline silicon solar cell (SSC) and a quantum dot intermediate band solar cell (QDIBSC). The basic structure of both grating equipped cell types is shown in Fig. 1. The diffraction grating is etched directly into the rear surface of the semiconductor wafer and is encased in a transparent cladding. The refractive index of the cladding material is taken to be $n_{\text {clad }}=1.52$, which corresponds roughly to $\mathrm{SiO}_{2}$ at the studied wavelengths. A planar perfect reflector is attached to the rear of the cladding material. Transmission of light through

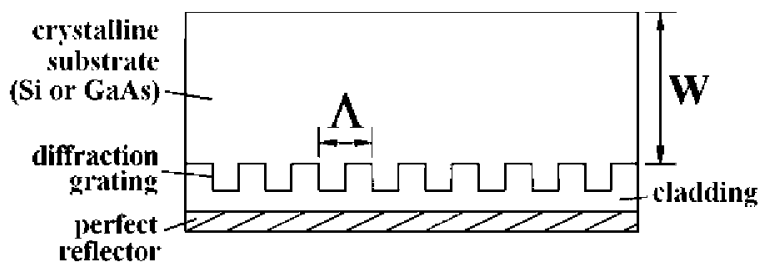

Fig. 1. Basic structure of solar cell with a diffraction grating on the rear face. the front surface of the solar cell is assumed to be perfect. It is assumed there is no absorption in the grating region. Only the laterally modulated grating region is modelled using RCWA. The unmodulated part of the cladding region and the perfect reflector are described, similarly to the cell, by an analytically generated matrix using the formalism in [6]. This implies the added assumption that the cladding region is thicker than the coherence length of the incident solar radiation ( $\approx 1 \mu \mathrm{m}$ ). It has been shown that thicker cladding regions are desirable due to reduced parasitic absorption in the rear reflector $[9,12]$.

The QD-IBSC has a stack of quantum dots (QDs) embedded in a thick crystalline wafer, usually GaAs, close to the front surface. The bound states of the QDs present an intermediate band (IB) between valence band $(\mathrm{VB})$ and conduction band $(\mathrm{CB})$ of the surrounding host material. In addition to the usual VB-CB transitions, a single carrier can also be photogenerated by two sub-bandgap photon induced transitions: VB-IB and IB-CB. The ideal detailed balance efficiency limit of an IB solar cell was found to be 63.2\% [13]. Each transition corresponds to a distinct sub-range of the incident illumination. The IB-CB transition is believed to have a weak absorption coefficient and therefore requires absorption enhancement for the corresponding wavelength range, which has been shown by electroluminescence measurements to be $1190 \mathrm{~nm}<\lambda<3350 \mathrm{~nm}$ for some QD-IBSCs [14]. It is assumed that the other transitions do not require absorption enhancement. The figure of merit for the QD-IBSC is therefore the photogenerated current corresponding to the IB-CB transition $J_{p h I B-C B}$. This is calculated by taking the integral in Eq. (1) over the $1190 \mathrm{~nm}<\lambda<3350 \mathrm{~nm}$ wavelength range. Although the substrate is some $200-300 \mu \mathrm{m}$ thick, IB-CB transitions only occur within the QD stack, whose thickness $w_{\text {stack }}$ depends on the number and thickness of QD layers. The absorption coefficient for IB-CB transitions is a subject of open research, and depends on the QD shape, dimensions and material and on the host material. For simplicity, we assume a wavelength independent absorption coefficient $\alpha_{I B-C B}$. A non-dispersive absorption coefficient is strictly unphysical since it violates a Kramers-Kronig relation; however, this assumption can be thought of as an approximation to an absorption coefficient that varies slowly over the $\mathrm{IB}-\mathrm{CB}$ wavelength range. Recent unpublished work has suggested an absorption coefficient of around $\alpha_{I B-C B}=60 \mathrm{~cm}^{-1}$, assuming a QD layer thickness of $80 \mathrm{~nm}$. For $20 \mathrm{QD}$ layers this would lead to a normal absorbance of $\alpha_{I B-C B} w_{\text {stack }}=0.01$. This value will be taken as the active layer normal absorbance unless otherwise mentioned. It is assumed that there is no parasitic free carrier absorption in the GaAs bulk. This implies that most of the substrate is undoped, with only thin doped layers above and below the QD stack to provide the pn junction. Consequentially the cell would need lateral contacts instead of a rear contact. This could be achieved in the same way the middle contact is often realized in monolithic, three-terminal tandem solar cells [15], which have been shown to lead to good efficiencies published in efficiency tables [16]. In any case, the results obtained here could be extended to include parasitic absorption by multiplying the calculated currents by the factor

\footnotetext{
$\alpha_{I B-C B} w_{\text {ODstack }}$

$\alpha_{I B-C B} w_{Q D s t a c k}+\alpha_{P} w_{P}$
}

where $\alpha_{P}$ is the absorption coefficient for any parasitic absorption and $w_{P}$ is the width of the layer in which such absorption takes place.

The SSC absorbing layer is modelled as a uniform slab of thickness $w$ and wavelength dependent absorption coefficient $\alpha_{s}(\lambda)$ taken from the data file supplied in PC1D [17]. The figure of merit for the SSC is the photogenerated current $J_{p h}$ calculated from Eq. (1) integrating over the entire AM1.5D spectral range. As transparent claddings are usually insulating, the cell would have to be point contacted at the rear. Otherwise, a transparent conductive oxide could be used as the cladding material. 
Using our method, the results of a single grating simulation can be used to calculate the absorption in cells with different absorption profiles. However, cells with different refractive indices require two distinct simulations of the grating because the scattering efficiencies and diffraction directions are refractive index dependent. To reduce computation time, a single wavelength-independent refractive index of $n_{\text {cell }}=3.33$ has been chosen for both cell types. This value is close to the refractive index of the QD-IBSC GaAs substrate at the wavelength range of interest, but somewhat distinct to that of silicon, for which band edge wavelengths are closer to $n=3.5$. We believe the resulting error should not be too extreme.

\subsection{Grating profiles}

The gratings under investigation consist of bi-periodic hexagonal arrays of elliptical binary wells and towers etched directly into the cell substrate. The well and tower profiles are shown in Fig. $2 \mathrm{~A}$ and $\mathrm{B}$, respectively. A bottom view of either grating is shown in Fig. $2 \mathrm{C}$. For both of the above cell types, optimisation is made of four key parameters: the grating period $\Lambda$, the well depth (tower height) $d$ and the well radii along the $x$ and $y$ axes: $r_{x}$ and $r_{y}$, respectively, (see Fig. 2). Each parameter is discussed in turn and comparison is made of the optimised parameters for each cell type allowing general design principles to be drawn.
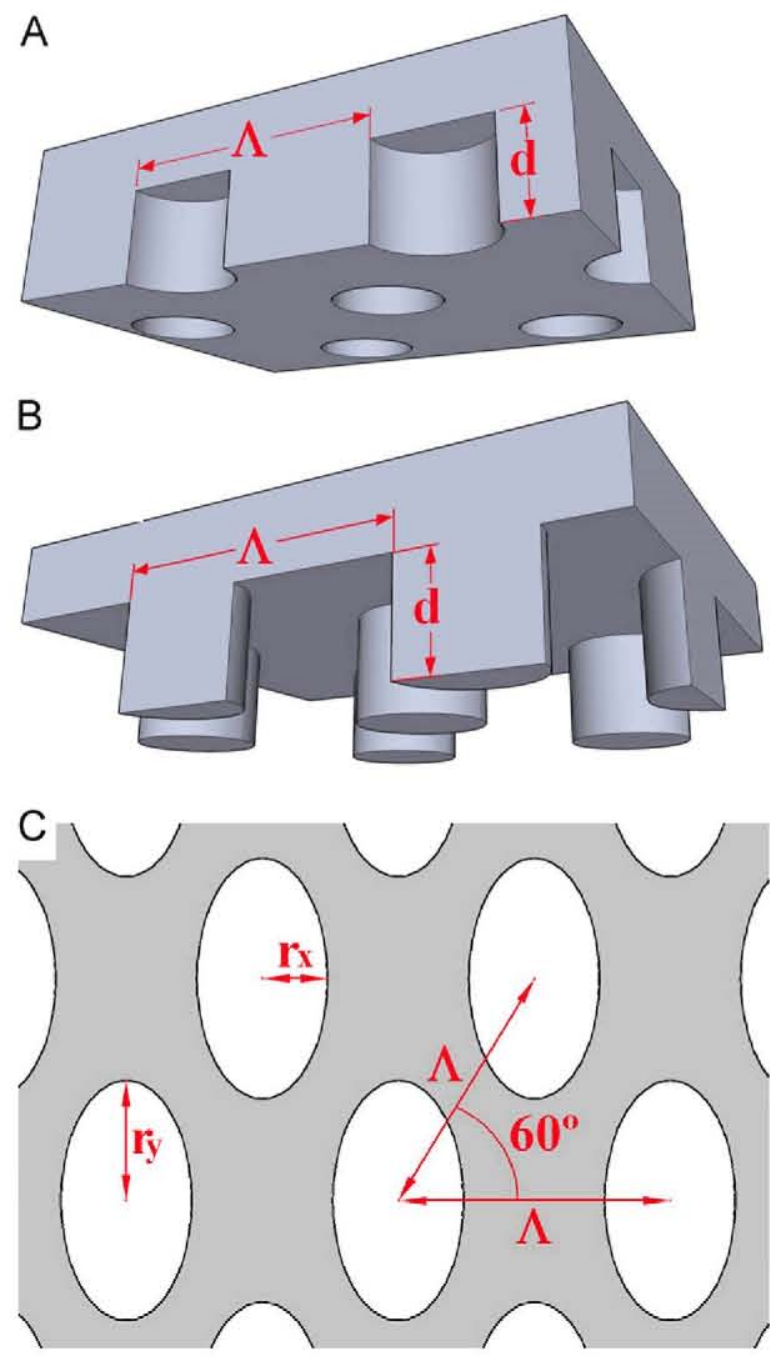

Fig. 2. (A) Profile of well grating. (B) Profile of tower grating. (C) Bottom view of either grating.

\subsection{Comparison to Lambertian trapping}

To assess the viability of diffraction gratings for light trapping, they must be compared with alternative light trapping mechanisms. Light trapping is most commonly realized by either rough [18] or geometric [19] texturing of the front or rear face of the solar cell. The theoretical mean optical path length enhancement caused by applying a perfectly Lambertian surface (one from which any incident illumination is reflected isotropically) is $4 n_{\text {cell }}^{2}$ : the well known Lambertian limit [20]. For extremely weakly absorbing media, this value can be directly equated to the absorption enhancement [21]; however, for media that absorb sufficiently to be of interest, the absorption enhancement for ideally Lambertian light trapping must be calculated using the formulae in Ref. [22]. Throughout this paper, the calculated absorption enhancements for diffraction gratings are compared to those for ideally Lambertian light trapping, calculated using the formulae in Ref. [22], and to the absorption in a cell equipped with only a planar back reflector. In both cases the idealising assumptions of perfect transmission at the front face and perfect reflection at the back face are assumed to allow fair comparison with the diffraction grating results. AboveLambertian light trapping is sought.

\subsection{Illumination conditions}

It is known that absorption enhancement above the Lambertian limit implicitly implies that the light trapping mechanism be angularly selective $[6,21,23]$. This means that above-Lambertian light trapping can only be achieved for light incident from some sub-set of the celestial hemisphere, absorption enhancement for light incident from outside this subset being necessarily belowLambertian. The simplest approach to above-Lambertian trapping is to seek absorption enhancement for incident light within a cone whose axis is normal to the solar cell and to apply a solar tracker to ensure that the solar disc remains within this cone. We assume an accurate solar tracker of acceptance angle $1^{\circ}$. The incident illumination is modelled as being isotropic within the corresponding cone (semi-angle $1^{\circ}$ ). Although the direct solar radiation at any given time is incident within a narrower cone (semi-angle $0.26^{\circ}$ ), the assumption of isotropic illumination within the acceptance cone can be thought of as the statistical mean contribution over the course of some period of time. The total intensity of the incident cone is taken to be the AM1.5D Direct Normal+Circumsolar spectrum. Incident light is considered unpolarised. Diffuse illumination is ignored. It should be noted that, if angle insensitive enhancement is desired (for stationary systems for example), the Lambertian limit is insurmountable and the ability of diffraction gratings to achieve better enhancement than random surface texturing is doubtful.

\section{Results}

\subsection{Period optimisation}

To understand the effect of the period, we begin by considering a diffraction grating applied to an absorbing medium that has wavelength independent absorption coefficient $\alpha$. The fraction of incident photons absorbed as a function of the incident wavelength to grating period ratio $\lambda / \Lambda$ is presented as solid curves in Fig. $3 \mathrm{~A}$ and $\mathrm{B}$ for absorbing media with normal absorbances of $\alpha w=0.0032$ and $\alpha w=0.032$, respectively. The diffraction grating is located on the rear surface and consists of a hexagonal array of circular towers with $r_{x}=r_{y}=0.37 \Lambda$ and $d=0.225 \Lambda$. In each figure, the dotted curve represents the $\lambda / \Lambda$ dependant upper limit to absorption using bi-periodic hexagonal gratings, calculated in 

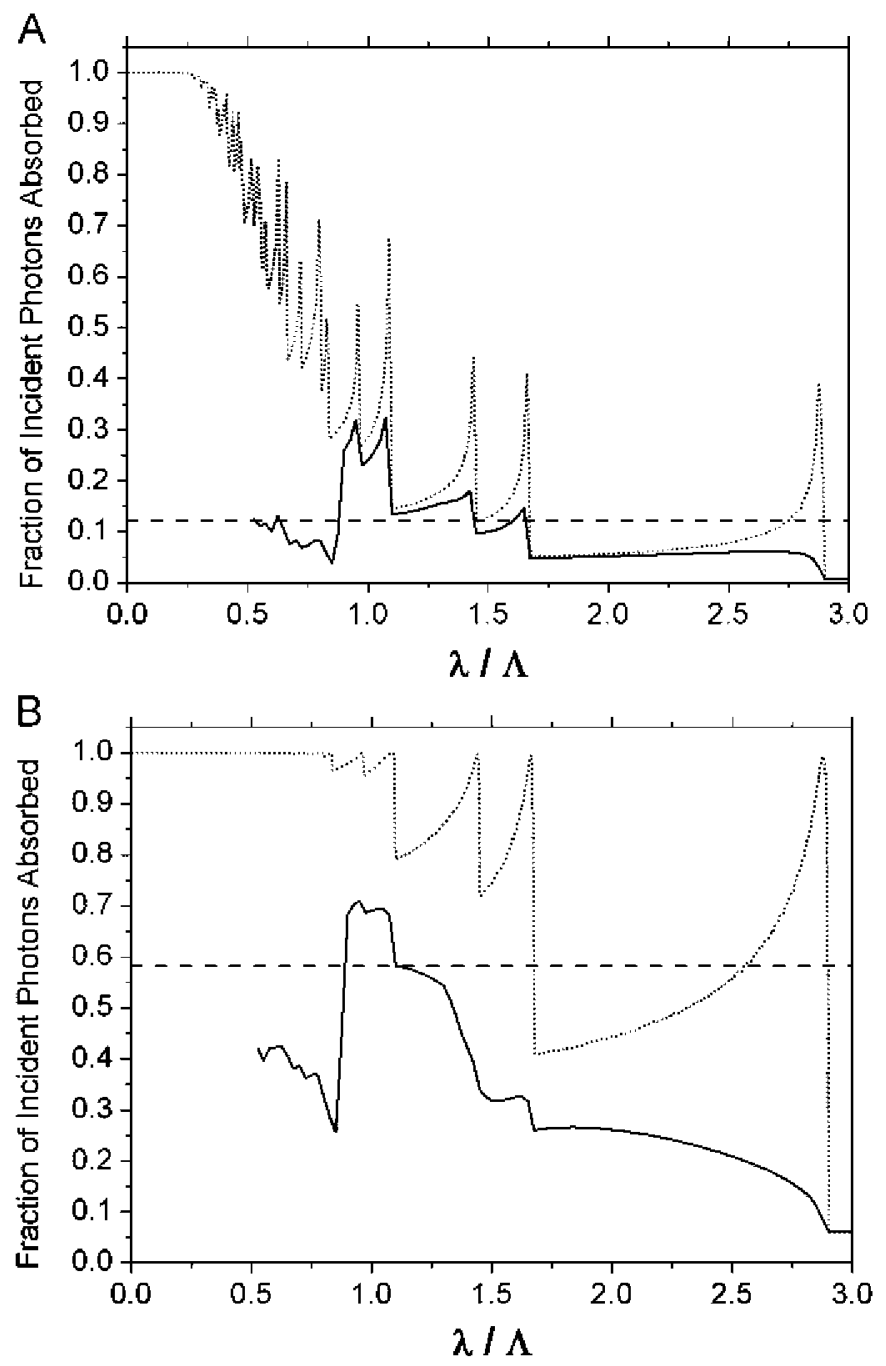

Fig. 3. Spectral fraction of incident photons absorbed in absorbing media with normal absorbance $\alpha w=0.0032$ (A) and $\alpha w=0.032$ (B) each equipped with a circular tower grating with $r_{x}=r_{y}=0.37 \AA$ and $d=2.25 A$.

Ref. [5], and the dashed horizontal line represents the calculated absorption for ideally Lambertian light trapping.

Beginning at the far right of Fig. $3 \mathrm{~A}$ and considering the solid curve, it can be seen that, on decreasing $\lambda / \Lambda$, a peak appears in the absorption each time a new oblique order is introduced, and tails off as the order becomes less obliquely inclined. There is an overall tendency for increased absorption with decreasing $\lambda / \Lambda$ as the angular extent of the cell becomes filled with diffracted cones. This continues until there is a sharp drop in the absorption at $\lambda / \Lambda \approx 0.9$. At this point, the lowest non zero-order diffracted cones enter the escape cone, leading to an increased coupling of light out of the cell and consequently a decrease in the internal brightness and hence the absorption. Due to computational constraints, calculations for $\lambda / A<0.5$ could not be made because, due to the high number of propagating diffracted orders, a large number of Fourier modes must be retained in the RCWA calculations; however, it is reasonable to assume that there would be a further drop when the next lowest order diffracted cones enter the escape cone at $\lambda / \Lambda \approx \sqrt{3 / 4} \approx 0.43$.

Fig. $3 \mathrm{~B}$ exhibits similar qualitative behaviour with the exception that the peaks are less well defined. As explained in Ref. [21], for weakly absorbing cells, the absorption is largely dominated by the geometrical mean optical path length, which, for $\lambda / A>0.9$, has been shown to be entirely dependent on the grating period and array geometry [6]. Conversely, for more strongly absorbing cells, there is a greater dependence on the coupling of the incident light to confined diffracted orders, which is largely dependent on the exact grating profile. This coupling is generally weak for very oblique orders, hence the rounding of the peaks.

Most importantly, it can be seen that, in both figures, the greatest absorption is achieved when $\lambda / A$ is just above the value at which diffracted cones begin to escape the cell. Theoretically, much higher absorption is possible for $\lambda / \Lambda<0.9$. As explained in [6], this can only be achieved by grating profiles, which restrict coupling between escaping and confined orders. None of the simple gratings studied in this work have accomplished this to any significant degree. We therefore conclude that, for simple gratings, the best light trapping is achieved in the $\lambda / A$ region in which the number of diffracted cones inside the medium is the highest; it may be without any non zero-order diffracted cones being able to escape the cell. This region can be roughly defined as $0.9<\lambda / \Lambda<1.1$, and easily remembered as being centred at the point at which the grating period is equal to the vacuum wavelength. It is reasonable to suggest that a similar argument could be made for diffraction gratings applied to thin film cells.

Since the refractive indices are taken to be wavelength independent, the simulations can be made in entirely dimensionless variables. This allows period optimisation to be made for a grating with defined dimensionless parameters using a single $\lambda / \boldsymbol{\Lambda}$ sweep without having to run a new set of RCWA calculations for each period.

\subsubsection{Period optimisation for the QD-IBSC}

Fig. 4 shows the calculated $J_{p h I B-C B}$ in a QD-IBSC with active layer normal absorbance of $\alpha_{I B-C B} w_{\text {stack }}=0.01$ for a circular tower grating with $r_{x}=r_{y}=0.37 A$ and $d=0.475 A$ as a function of the period. Two distinct peaks can be seen at $A=1330 \mathrm{~nm}$ and $A=1650 \mathrm{~nm}$, the latter being the highest. For these optimum periods, the spectral number of absorbed photons and spectral fraction of incident photons absorbed are plotted in Fig. $5 \mathrm{~A}$ and $\mathrm{B}$, respectively. The blue and red curves correspond to $A=1650 \mathrm{~nm}$ and $A=1330 \mathrm{~nm}$, respectively. The black curve in Fig. $5 \mathrm{~A}$ is the spectral photon flux of the AM1.5D spectrum. It can be seen that the optimum periods are simply those, which align the aforementioned $0.9<\lambda / \Lambda<1.1$ region with the predominant peaks in the AM1.5D spectrum that lie within the IB-CB wavelength range. For this grating, the highest integrated photon absorption is for

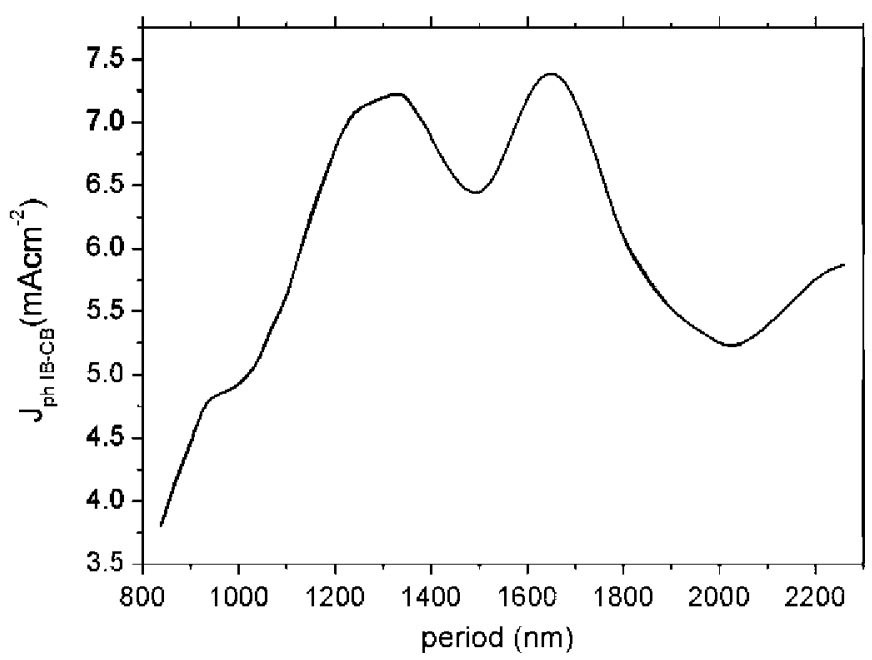

Fig. 4. Photogenerated current $J_{p h I B-C B}$ in a QD-IBSC with $\alpha_{I B-C B} w_{s t a c k}=0.01$ as a function of the grating period $A$. Circular tower grating with $r_{x}=r_{y}=0.37 \mathrm{~A}$ and $d=0.475 A$. 


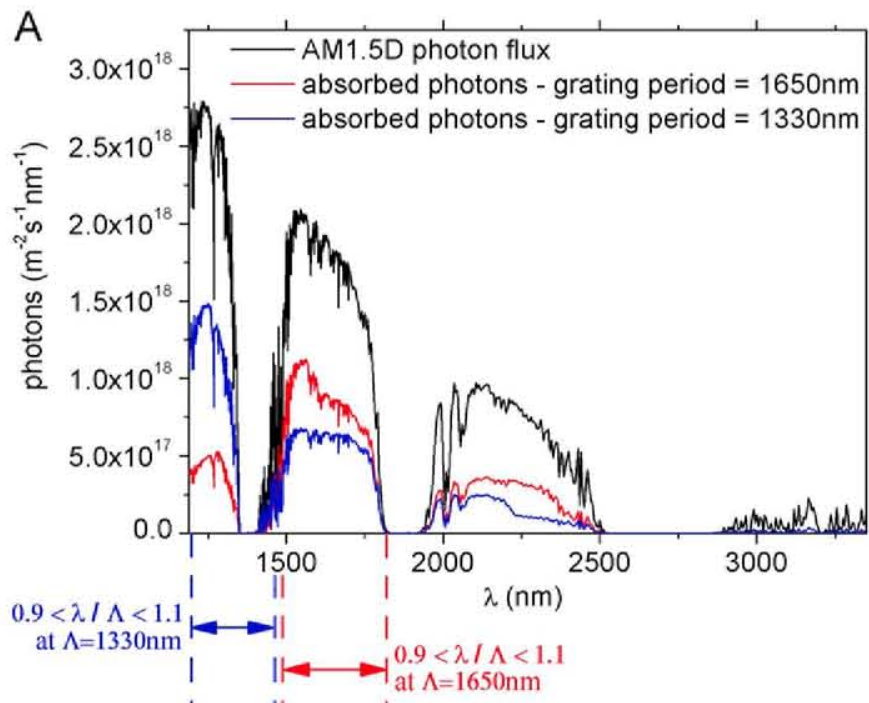

$\mathrm{B}$

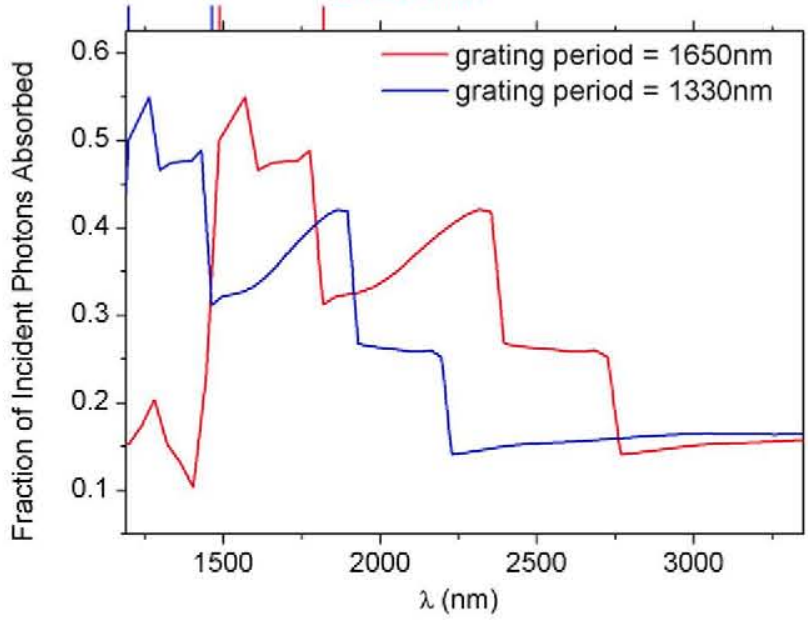

Fig. 5. (A) Spectral AM1.5D photon flux (black curve) and the number of photons absorbed in a QD-IBSC with $\alpha_{I B-C B} W_{\text {stack }}=0.01$ for a grating with period $1650 \mathrm{~nm}$ (red curve) and $1330 \mathrm{~nm}$ (blue curve). (B) The spectral fraction of incident photons absorbed for the same periods. All circular tower gratings with $r_{x}=r_{y}=0.37 \AA$ and $d=0.475 \AA$. (For interpretation of the references to colour in this figure legend, the reader is referred to the web version of this article.)

$\Lambda=1650 \mathrm{~nm}$ (Fig. 4); however, depending on the exact grating profile, the $\Lambda=1330 \mathrm{~nm}$ peak is sometimes higher.

\subsubsection{Period optimisation for the silicon solar cell}

Fig. 6 shows the number of photons absorbed in the SSC for a circular tower grating with $r_{x}=r_{y}=0.37 \Lambda$ and $d=0.225 \Lambda$ as a function of the period. There is a single peak at $\Lambda=1080 \mathrm{~nm}$. The thick blue and red curves in Fig. 7 (right scale) show the spectral fraction of absorbed photons near the silicon band edge for a grating of optimum period $(\Lambda=1080 \mathrm{~nm})$ and for a cell with only a planar back reflector, respectively. The thin lines of the same colours show the spectral number of absorbed photons and the thin black line shows the photon flux of the AM1.5D spectrum (left scale). The optimum period aligns the $0.9<\lambda / \Lambda<1.1$ range with the wavelength range where absorption in the SSC cell is weak but non-zero, slightly right shifted to take advantage of the peak in the AM1.5D spectrum.

\subsection{Depth optimisation}

In the following section, the grating periods are fixed to the above mentioned optimum values (1650 $\mathrm{nm}$ for the QD-ISBC and $1080 \mathrm{~nm}$ for the silicon cell, respectively) and the well and tower

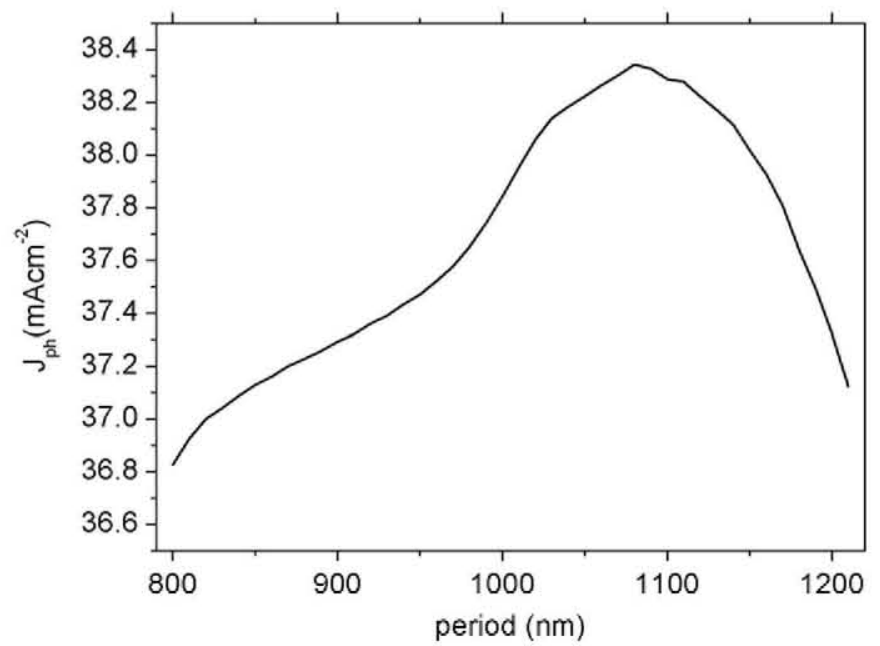

Fig. 6. Photogenerated current $J_{p h}$ in a $40 \mu \mathrm{m}$ thick SSC as a function of the grating period $A$. Circular tower grating with $r_{x}=r_{y}=0.37 \AA$ and $d=0.225 \AA$.

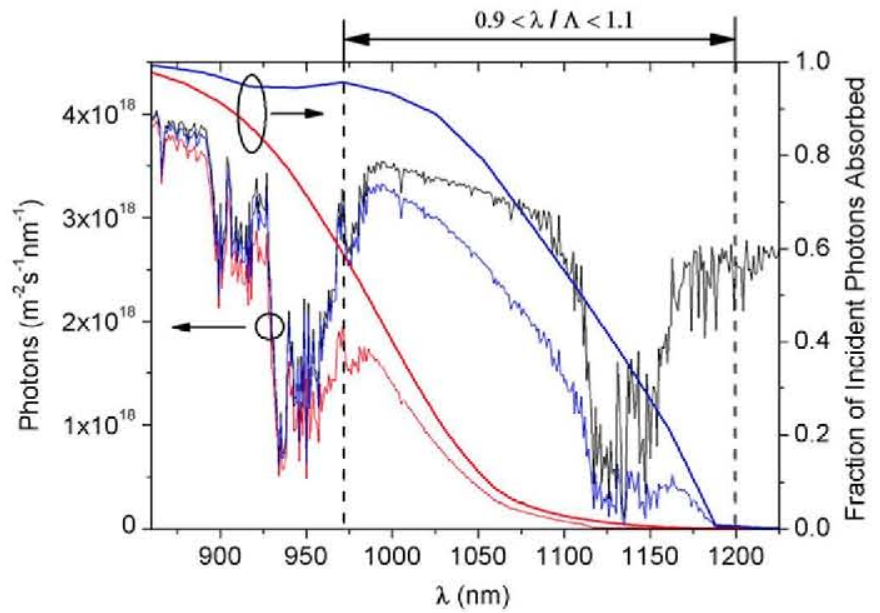

Fig. 7. Right scale: the spectral fraction of absorbed photons for a grating of optimum period ( $\Lambda=1080 \mathrm{~nm}$ ) (thick blue curve) and for a cell with only a planar back reflector (thick red curve). Left scale: the spectral AM1.5D photon flux (thin black curve) and the number of absorbed photons for a grating of optimum period (thin blue curve) and for a cell with only a planar back reflector (thin red curve). Grating is a circular tower grating with $r_{x}=r_{y}=0.37 \Lambda$ and $d=0.225 \Lambda$. (For interpretation of the references to colour in this figure legend, the reader is referred to the web version of this article.)

depths are varied. By fixing the period, the $0.9<\lambda / \Lambda<1.1$ range is fixed to the wavelength range in which light trapping is most beneficial. It will be seen that the optimum depths are those, which maximise the absorption within this range.

Fig. 8 shows the generated IB-CB photocurrent in the grating equipped QD-IBSC as a function of the grating depth $d$ normalised to units of the grating period $\Lambda$. The red and black curves are for circular wells and towers, respectively, each with duty cycles of $50 \%$ (i.e. the well or tower area is half the total area). The same is shown in Fig. 9 for the SSC.

The curves are characterised by periodic oscillations at intervals of $\Delta d / \Lambda \approx 0.55$, modulated by a higher frequency oscillation at intervals of $\Delta d / \Lambda \approx 0.15$. These oscillations occur at similar period normalised depths for each cell and grating type. We postulate that this behaviour is largely dependent on the scattering efficiencies coupling the incident solar radiation to the zero-order reflected and transmitted diffraction orders (i.e. the specular reflection and transmission for vertical incidence). Light coupled into the reflected zero-order is coupled out of the cell 


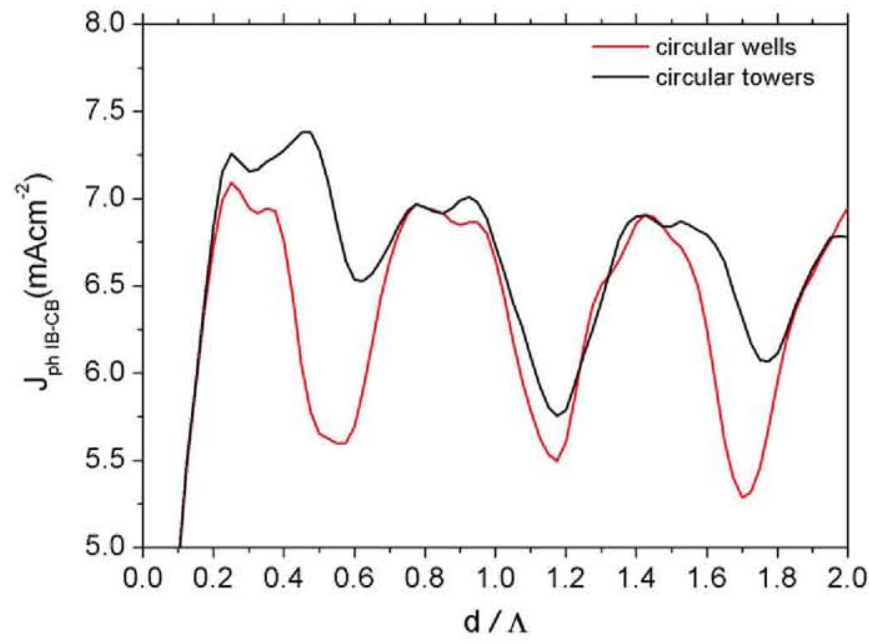

Fig. 8. Photogenerated current $J_{p h I B-C B}$ in a QD-IBSC with $\alpha_{I B-C B} w_{\text {stack }}=0.01$ as a function of the period normalised grating depth $d / \Lambda$. Circular well (black curve) and tower (red curve) gratings with $r_{x}=r_{y}=0.37 \Lambda$ and $A=1650 \mathrm{~nm}$. (For interpretation of the references to colour in this figure legend, the reader is referred to the web version of this article.)

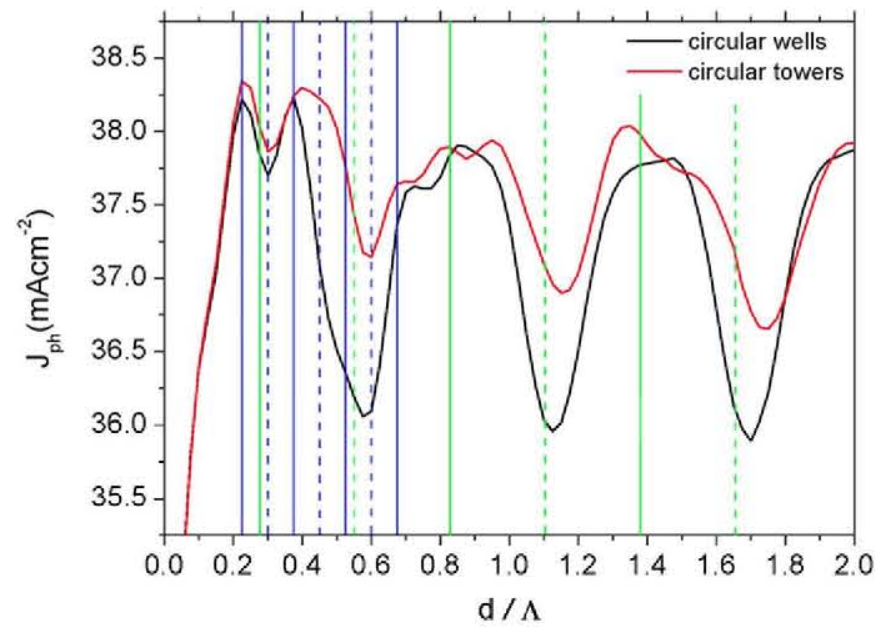

Fig. 9. Photogenerated current $J_{p h}$ in a $40 \mu \mathrm{m}$ thick SSC as a function of the period normalised grating depth $d / \Lambda$. Circular well (black curve) and tower (red curve) gratings with $r_{x}=r_{y}=0.37 \Lambda$ and $\Lambda=1080 \mathrm{~nm}$. Vertical lines represent depths at which the $0.9<\lambda \mid \Lambda<1.1$ region coincides with specular reflection minima (solid blue) and maxima (dashed blue) and with specular transmission minima (solid green) and maxima (dashed green). (For interpretation of the references to colour in this figure legend, the reader is referred to the web version of this article.)

only after a double pass and light coupled into the transmitted zero-order is reflected at the rear reflector and re-transmitted into the upward travelling zero-order with the same efficiency due to reciprocity, again escaping after a double pass. It is therefore desirable to minimise coupling of the incident light into both of these orders.

The wavelength dependence of the scattering efficiencies with which normally incident light is coupled into the reflected (black curve) and transmitted (red curve) zero-orders is plotted in Fig. $10 \mathrm{~A}$ and B for circular well gratings of depth $d=0.225 A$ and $d=1.7 \Lambda$, respectively, (these are the optimum and antioptimum depths from Fig. 9). It can be seen in both figures that the zero-order efficiencies exhibit minima and maxima corresponding to Fabry-Perot interferences between waves reflected (or transmitted) at the two surfaces of the binary grating. The aforementioned $0.9<\lambda / \Lambda<1.1$ range where the best light trapping is achieved is shown in the figures. It can be seen that for $d=0.225 \Lambda$ (Fig. 10A) this region is aligned with a specular
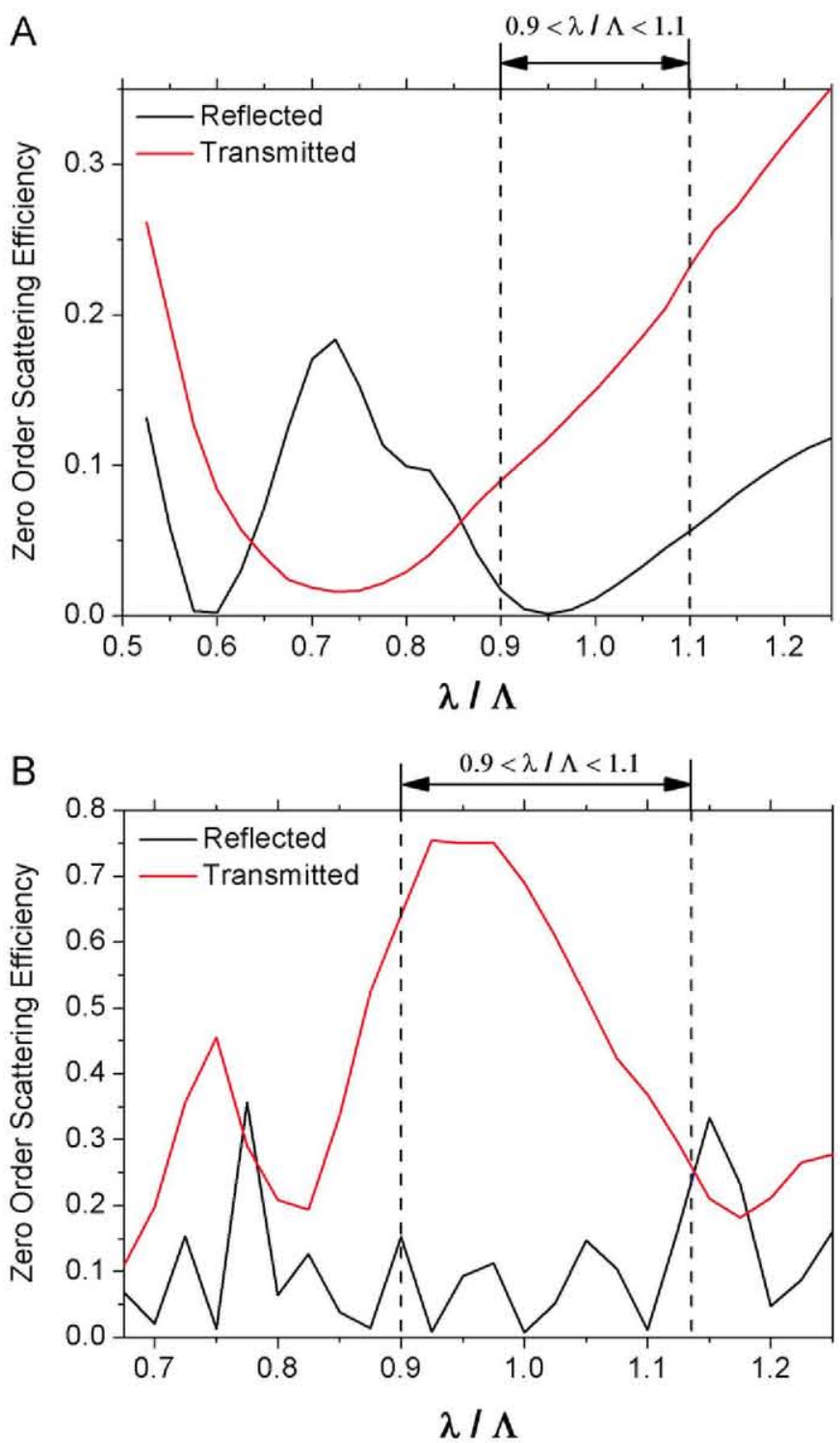

Fig. 10. Scattering efficiency with which vertically incident light couples to the reflected (black curve) and transmitted (red curve) zero-order (i.e. specular transmission and reflection). Circular well gratings with $r_{x}=r_{y}=0.37 \Lambda$. $(A): d=0.225 \Lambda$ and $(B): d=1.7 \AA$. (For interpretation of the references to colour in this figure legend, the reader is referred to the web version of this article.)

reflection minimum, leading to the peak at this depth in Fig. 9; whereas for $d=1.7 \mathrm{~A}$ (Fig. 10B) this region is aligned with a strong specular transmission maximum, leading to the nadir at this depth in Fig. 9. In general, the normalised well depths $(d / \Lambda)_{r-}$ and $(d / \Lambda)_{r+}$ at which spectral reflection minima and maxima align, respectively, with the $0.9<\lambda / \Lambda<1.1$ region (taking the centre of the region to be $\lambda / \Lambda=1$ ) are
$(d / \Lambda)_{r-}=\frac{m-1 / 2}{2 n_{\text {cell }}}$
$(d / \Lambda)_{r+}=\frac{m}{2 n_{\text {cell }}}$

For spectral transmission minima and maxima these are

$(d / \Lambda)_{t-}=\frac{m-1 / 2}{n_{\text {cell }}-n_{\text {clad }}}$
$(d / \Lambda)_{r+}=\frac{m}{n_{\text {cell }}-n_{\text {clad }}}$ 
where $m$ is an integer. For certain values of $m$, the vertical blue lines in Fig. 9 show $(d / \Lambda)_{r_{-}}$(solid) and $(d / \Lambda)_{r_{-}}$(dashed) and the vertical green lines show $(d / \Lambda)_{t-}$ (solid) and $(d / \Lambda)_{t+}$ (dashed). Good agreement with the bi-frequencial oscillations is shown. For deep gratings, there are many Fabry-Perot interference peaks and troughs within the $0.9<\lambda / \Lambda<1.1$ region, which cancel out each other (see Fig. 10B). Hence the higher frequency oscillations in Figs. 8 and 9 become less pronounced and less regular for deeper gratings. For both cell types the optimum gratings are shallow: $d=0.45 \Lambda$ for the QD-IBSC and $d=0.225 \Lambda$ for the SSC.

\subsection{Optimisation of lateral dimensions}

In this section the normalised well depth is fixed at $d / \Lambda=0.25$ and $r_{x}$ and $r_{y}$ are varied leading to elliptical wells and towers. Instead of fixing the period at a certain value, the period has been optimised for each $\left(r_{x} / \Lambda, r_{y} / \Lambda\right)$ pair. Using our simulation method, the extra computational time required to do this is minimal, as explained in section 3.1.

For the QD-ISBC, $J_{p h I B-C B}$ is shown as a function of the period normalised radii in Fig. $11 \mathrm{~A}$ and $\mathrm{B}$ for elliptical well and tower gratings, respectively. For the silicon cell, $J_{p h}$ is shown in Fig. $12 \mathrm{~A}$ and B for elliptical well and tower gratings, respectively. In each graph, the black cross shows the optimum radii, and the blue line represents the locus of radii for which the grating has a duty cycle of $50 \%$. Unlike for the grating depth, the optimum values do not occur at the same radii for each cell and grating type. The shared characteristics of the four graphs are that the optima occur for gratings with duty cycles close to $50 \%$, in agreement with similar results for thin-film applications [24,25], and that they occur for wells, which are not extremely narrow in either dimension. The radii dependence is generally weaker than the height dependence and the tolerance is correspondingly high.
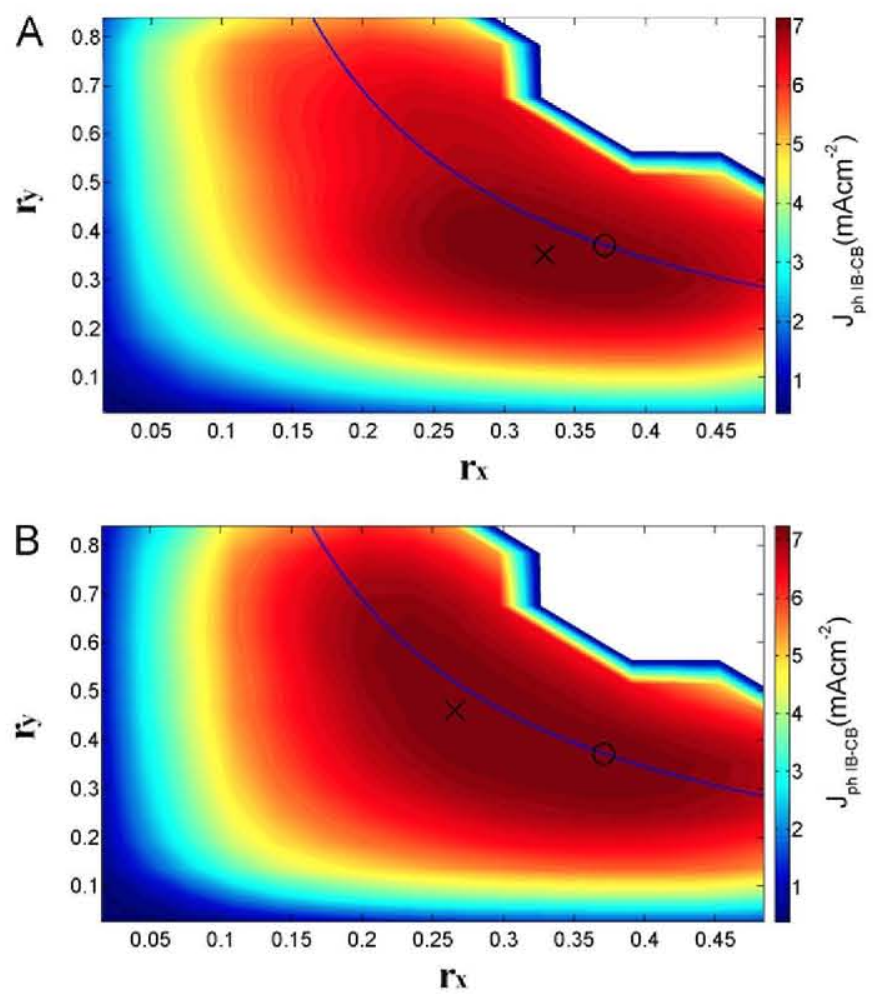

Fig. 11. Photogenerated current $J_{p h I B-C B}$ in a QD-IBSC with $\alpha_{I B-C B} w_{\text {stack }}=0.01$ as a function of the period normalised well or tower radii $\left(r_{x} / \Lambda, r_{y} / \Lambda\right) \cdot \mathrm{d}=0.225 \Lambda$ and the period has been optimised for each $\left(r_{x} / \Lambda, r_{y} / \Lambda\right)$ pair. (A): well grating. (B): tower grating.
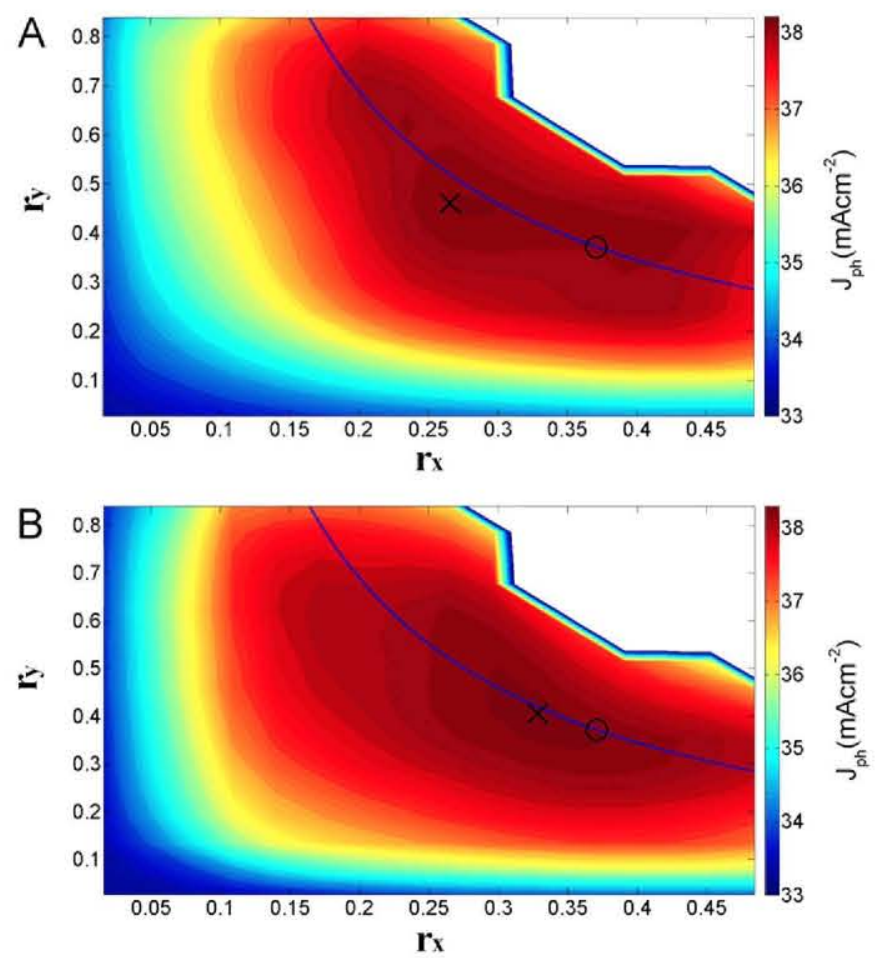

Fig. 12. Photogenerated current $J_{p h}$ in a $40 \mu \mathrm{m}$ thick SSC as a function of the period normalised well or tower radii $\left(r_{x} / \Lambda, r_{y} / \Lambda\right) . d=0.225 \Lambda$ and the period has been optimised for each $\left(r_{x} \mid \Lambda, r_{y} / \Lambda\right)$ pair. (A): well grating. (B): tower grating.

The black circle in each graph shows the $\left(r_{x} / \Lambda, r_{y} / \Lambda\right)$ pair corresponding to a circular well or tower with a duty cycle of $50 \%$ (i.e. $r_{x}=r_{y}=0.37 \AA$ ). In all cases studied, this leads to a photogenerated current that is within $1 \%$ of the optimum for the QD-IBSC and within $0.1 \%$ of the optimum for the SSC. It is concluded that elliptical towers or wells lead to no significant improvement over simple circular towers or wells.

\subsection{Optimisation for $Q D$-IBSC cells with different numbers of $Q D$ layers and SSC cells with different thicknesses}

The aforementioned grating parameters have been optimised for QD-IBSCs with a range of normal absorbances and for SSCs with a range of thicknesses. For the QD-IBSC, the normal absorbance has been translated into the number of QD layers assuming an absorption coefficient of $\alpha_{I B-C B}=60 \mathrm{~cm}^{-1}$ and a QD layer thickness of $80 \mathrm{~nm}$. In all studied cases, optimisation of the lateral radii $r_{x}$ and $r_{y}$ provided an improvement of less than $1 \%$ for the QD-IBSC and less than $0.1 \%$ for the SSC compared to the simple circular pattern with a duty cycle of $50 \%$. Furthermore, currents calculated for tower gratings were consistently higher than those calculated for well gratings. Hence all results presented in this section are for circular towers with $r=0.37 \Lambda$.

Table 1 shows the optimised parameters for QD-IBSCs with a range of numbers of $\mathrm{QD}$ layers along with the resulting photogenerated current. The well depth is normalised to units of the optimum grating period. The total available number of photons in the AM1.5D spectrum in the $1190 \mathrm{~nm}<\lambda<3350 \mathrm{~nm}$ interval is $1.29 \times 10^{21} \mathrm{~cm}^{-2} \mathrm{~s}^{-1}$ implying a maximum possible photocurrent of $20.6 \mathrm{mAcm}^{-2}$. The optimised parameters for $20 \mathrm{QD}$ layers shown in Table 1 are those for which data is presented in Figs. 4 and 5 .

Fig. 13 shows graphically the $J_{p h I B-C B}$ for the optimised gratings (blue curve) compared to that for a planar reflector (black curve) and for an ideally Lambertian reflector (red curve), calculated 
Table 1

Optimised grating parameters and resulting photogenerated current for QD-IBSCs with a range of numbers of QD layers. All circular tower gratings with $r_{x}=r_{y}=0.37 \AA$.

\begin{tabular}{cllc}
\hline $\begin{array}{l}\text { Number of } \\
\text { QD layers }\end{array}$ & $\begin{array}{l}\Lambda \\
(\mu \mathrm{m})\end{array}$ & $d / \Lambda$ & $\begin{array}{c}J_{\text {ph IB-CB }} \\
\left(\mathrm{mAcm}^{-2}\right)\end{array}$ \\
\hline 20 & 1650 & 0.45 & 7.4 \\
66 & 1340 & 0.425 & 12.0 \\
200 & 1330 & 0.425 & 15.9 \\
660 & 1320 & 0.425 & 18.7 \\
2000 & 1320 & 0.425 & 20.3 \\
\hline
\end{tabular}

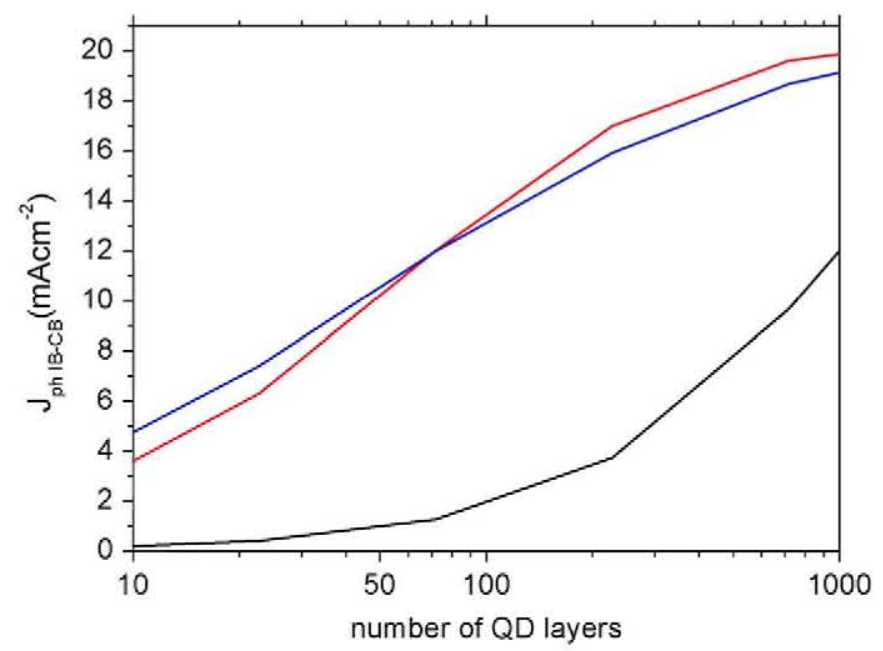

Fig. 13. Photogenerated current $J_{p h I B-C B}$ in a QD-IBSC equipped with the optimised gratings (blue curve). For comparison, the $J_{p h} I B-C B$ is shown for the same cell equipped with an ideally Lambertian back reflector (red curve) and with a planar back reflector (black curve). (For interpretation of the references to colour in this figure legend, the reader is referred to the web version of this article.)

using the formulae in Ref. [22]. For cells with less than 70 QD layers, the calculated $J_{p h I B-C B}$ for the optimised gratings are above that of the ideally Lambertian scatterer. QD-IBSCs fabricated to date have up to 50 QD layers [26] and therefore lie within this range. However, it must be noted that for $50 \mathrm{QD}$ layers only half of the available photons are absorbed. To approach full absorption of the available photons and hence approach the maximum possible IB-CB photocurrent, it will be necessary to either improve light trapping beyond what is presented in this paper or increase the number of QD layers.

Table 2 shows the optimised parameters for SSCs with a range of thicknesses along with the resulting $J_{p h}$. The optimum period is larger for thicker cells because the wavelength range where absorption in the SSC cell is weak becomes more closely packed towards the band edge. The optimum normalised depth remains close to $d=0.25 \Lambda$. The optimised parameters for SSCs of thickness $40 \mu \mathrm{m}$ shown in Table 2 are those for which data is presented in Figs. 6 and 7.

Fig. 14 shows graphically the $J_{p h}$ for the optimised gratings (blue curve) compared to that for a planar reflector (black curve) and for an ideally Lambertian reflector (red curve). The calculated $J_{p h}$ s for the optimised gratings are consistently above that of the ideally Lambertian scatterer for cells with thicknesses of $10 \mu \mathrm{m}$ and above. The calculated $J_{p h}$ for a $20 \mu \mathrm{m}$ SSC equipped with the optimised grating is higher than that of a $200 \mu \mathrm{m}$ SSC with a planar back reflector.
Table 2

Optimised grating parameters and resulting photogenerated current for SSCs with a range of thicknesses. All circular tower gratings with $r_{x}=r_{y}=0.37 \Lambda$.

\begin{tabular}{rlll}
\hline $\begin{array}{l}\text { Cell thickness } \\
(\mu \mathrm{m})\end{array}$ & $\begin{array}{l}A \\
(\mu \mathrm{m})\end{array}$ & $d / \Lambda$ & $\begin{array}{l}J_{p h} \\
\left(\mathrm{mAcm}^{-2}\right)\end{array}$ \\
\hline 1 & 740 & 0.25 & 27.6 \\
5 & 990 & 0.225 & 33.6 \\
10 & 1020 & 0.225 & 35.7 \\
20 & 1020 & 0.25 & 37.2 \\
30 & 1080 & 0.225 & 37.9 \\
40 & 1080 & 0.225 & 38.3 \\
50 & 1080 & 0.225 & 38.6 \\
75 & 1080 & 0.25 & 39.1 \\
100 & 1090 & 0.25 & 39.4 \\
200 & 1110 & 0.25 & 40.0 \\
300 & 1120 & 0.25 & 40.3 \\
\hline
\end{tabular}

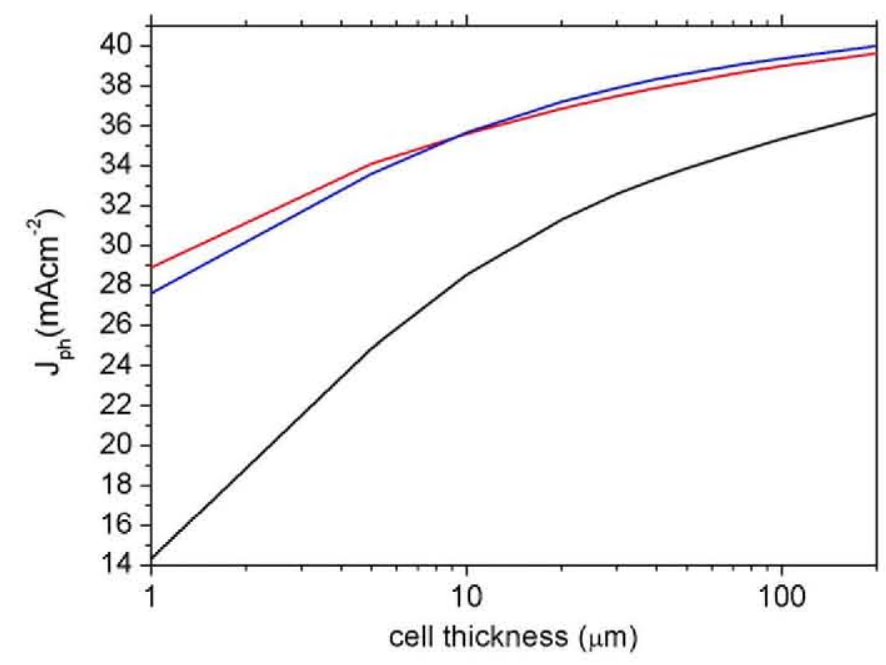

Fig. 14. Photogenerated current $J_{p h}$ in a SSC equipped with the optimised gratings (blue curve). For comparison, the $J_{p h I B-C B}$ is shown for the same cell equipped with an ideally Lambertian back reflector (red curve) and with a planar back reflector (black curve). (For interpretation of the references to colour in this figure legend, the reader is referred to the web version of this article.)

\section{Conclusions}

Bi-periodic binary gratings consisting of hexagonal arrays of elliptical wells and towers have been computationally studied and optimised for application to absorption enhancement in a QD-IBSC and in a thin SSC. It is found that, for these structures, the range of wavelength to grating period ratios at which absorption enhancement is highest is that at which the number of diffracted cones inside the cell is the highest; it may be without any non zero-order diffracted cones being able to escape the cell, roughly corresponding to $0.9<\lambda / \Lambda<1.1$. The optimum period aligns this range with the wavelength range for which light trapping is most beneficial, i.e. where the incident photon flux is high and the material absorption is low but nonzero. The optimum heights are those, which align the $0.9<\lambda / \Lambda<1.1$ range with minima in the reflected and transmitted zero-order scattering efficiencies, which exhibit oscillations of a Fabry-Perot nature. Optimisation of the well or tower radii along both ellipse axes leads to slightly distinct optimum values for each grating type and cell type. However, for all cases studied, it is found that a circular well or tower with a duty cycle of $50 \%$ (i.e. $r_{x}=r_{y}=0.37 \Lambda$ ) leads to a photogenerated current that is within $1 \%$ of the optimum for the QD-IBSC and within $0.1 \%$ of the optimum for the SSC. Tower gratings lead to 
greater absorption enhancement than well gratings for all cases studied.

The optimised grating parameters and resulting photogenerated currents have been presented for QD-IBSCs with a range of QD layers and for SSCs with a range of thicknesses. With QD-IBSCs with less than $70 \mathrm{QD}$ layers, the optimised gratings lead to higher photogenerated current than does an ideally Lambertian scatterer. However, it is also concluded that, to absorb a substantial fraction of the available photons it will be necessary to either improve light trapping beyond what is presented in this paper or increase the number of QD layers above what has been grown to date (up to 50 layers). For SSCs, the optimised gratings consistently lead to above-Lambertian enhancement for cells with thicknesses of $10 \mu \mathrm{m}$ or greater. The calculated photogenerated current for a $20 \mu \mathrm{m}$ SSC equipped with the optimised grating is higher than that of a $200 \mu \mathrm{m}$ SSC with a planar back reflector.

\section{Acknowledgements}

This work was supported by the European Commission project IBPOWER (Contract 211640), the Spanish Science Ministry projects NANOGEFFES (ENE2009-14481-C02-01) and DENQUIBAND (PLE2009-0045). Alexander Mellor gratefully acknowledges the Comunidad de Madrid for financial support through the scholarship Personal Investigador de Apoyo.

\section{References}

[1] C. Heine, R.H. Morf, Submicrometer gratings for solarenergy applications, Applied Optics 34 (1995) 2476-2482.

[2] J.H. Zhao, A.H. Wang, P. Campbell, M.A. Green, A 19.8\% efficient honeycomb multicrystalline silicon solar cell with improved light trapping, IEEE Transactions on Electron Devices 46 (1999) 1978-1983.

[3] H. Sai, Y. Kanamori, K. Arafune, Y. Ohshita, M. Yamaguchi, Light trapping effect of submicron surface textures in crystalline Si solar cells, Progress in Photovoltaics 15 (2007) 415-423.

[4] M. Kroll, S. Fahr, C. Helgert, C. Rockstuhl, F. Lederer, T. Pertsch, Employing dielectric diffractive structures in solar cells-a numerical study, Physica Status Solidi A-Applications and Materials Science 205 (2008) 2777-2795.

[5] I. Tobias, A. Luque, A. Marti, Light intensity enhancement by diffracting structures in solar cells, Journal of Applied Physics 104 (2008) 034502

[6] A. Mellor, I. Tobías, A. Martí, M. Mendes, A. Luque, Upper limits to absorption enhancement in thick solar cells using diffraction gratings, Progress in Photovoltaics: Research and Applications 19 (2011) 676-678.

[7] C. Kyoung Seok, P. Mandal, K. Kyounghyun, B. In Hyung, L. Sangjun, L. Hanjo, C. Doo Jin, K. Sangin, L. Jaejin, F. Rotermund, Improved efficiency in GaAs solar cells by $1 \mathrm{D}$ and 2D nanopatterns fabricated by laser interference lithography, Optics Communications 284 (2011) 2608-2612.

[8] S.Y. Chou, P.R. Krauss, P.J. Renstrom, Imprint lithography with 25-nanometer resolution, Science 272 (1996) 85-87.

[9] M. Peters, M. Ruediger, B. Blaesi, W. Platzer, Electro-optical simulation of diffraction in solar cells, Optics Express 18 (2010) A584-A593.

[10] M. Neviere, E. Popov, Light Propagation in Periodic Media: Differential Theory and Design (Optical Engineering), Marcell Dekker, New York, 2002.

[11] M. Born, E. Wolf, Principles of Optics: Electromagnetic Theory of Propagation, Interference and Diffraction of Light, 7th Edition, Cambridge University Press, Cambridge, 1999 , pp. 352-359.

[12] P. Berger, H. Hauser, D. Suwito, S. Janz, M. Peters, B. Blaesi, M. Hermle, Realization and evaluation of diffractive systems on the back side of silicon solar cells, in: R.B. Wehrspohn, Andreas Gombert (Eds.), Photonics for Solar Energy Systems III, Proc. of SPIE 722504.

[13] A. Luque, A. Marti, Increasing the efficiency of ideal solar cells by photon induced transitions at intermediate levels, Physical Review Letters 78 (1997) 5014-5017.

[14] A. Luque, A. Marti, C. Stanley, N. Lopez, L. Cuadra, D. Zhou, J.L. Pearson, A. McKee, General equivalent circuit for intermediate band devices: Potentials, currents and electroluminescence, Journal of Applied Physics 96 (2004) 903-909.

[15] MW Wanlass, T J Coutts, JS. Ward, KA Emery, T A Gessert CR Osterwald, IEEE, Advanced high-efficiency concentrator tandem solarcells, in: Conference Record of the Twenty Second IEEE Photovoltaic Specialists Conference1991, Vols 1 and 2, 1991, pp. 38-45.

[16] M.A. Green, K. Emery, D.L. King, Y. Hisikawa, W. Warta, Solar cell efficiency tables (Version 27), Progress in Photovoltaics 14 (2006) 45-51.

[17] D.A. Clugston, P.A. Basore, IEEE, PC1D version 5: 32-bit solar cell modeling on personal computers, in: Conference Record of the Twenty Sixth IEEE Photovoltaic Specialists Conference-1997, 1997, pp. 207-210.

[18] C. Rockstuhl, S. Fahr, K. Bittkau, T. Beckers, R. Carius, F.J. Haug, T. Soederstroem, C. Ballif, F. Lederer, Comparison and optimization of randomly textured surfaces in thin-film solar cells, Optics Express 18 (2010) A335-A342.

[19] P. Campbell, M.A. Green, Light trapping properties of pyramidally textured surfaces, Journal of Applied Physics 62 (1987) 243-249.

[20] E. Yablonovitch, G.D. Cody, Intensity enhancement in textured optical sheets for solar-cells, IEEE Transactions on Electron Devices 29 (1982) 300-305.

[21] J.C. Miñano, Optical Confinement in Photovoltaics, in: A. Luque, G. Araujo (Eds.), Physical Limitations to Photovoltaic Energy Conversion, Adam Hilger, Bristol, 1990 , pp. $50-55$

[22] M.A. Green, Lambertian light trapping in textured solar cells and lightemitting diodes: Analytical solutions, Progress in Photovoltaics 10 (2002) $235-241$.

[23] Z. Yu, S. Fan, Angular constraint on light-trapping absorption enhancement in solar cells, Applied Physics Letters 98 (2011) 011106.

[24] N.-N. Feng, J. Michel, L. Zeng, J. Liu, C.-Y. Hong, L.C. Kimerling, X. Duan, Design of highly efficient light-trapping structures for thin-film crystalline silicon solar cells, IEEE Transactions on Electron Devices 54 (2007) 1926-1933.

[25] L. Zhao, Y.H. Zuo, C.L. Zhou, H.L. Li, H.W. Diao, W.J. Wang, A highly efficient light-trapping structure for thin-film silicon solar cells, Solar Energy 84 (2010) $110-115$.

[26] D. Alonso-Alvarez, A.G. Taboada, J.M. Ripalda, B. Alen, Y. Gonzalez, L. Gonzalez, J.M. Garcia, F. Briones, A. Marti, A. Luque, A.M. Sanchez, S.I. Molina, Carrier recombination effects in strain compensated quantum dot stacks embedded in solar cells, Applied Physics Letters 93 (2008) 123114. 